formerly known as anti-p155), which has been shown to be useful in the diagnosis of cancerassociated myositis. ${ }^{2,3}$ In the reported case, negativity for this antibody would imply that the improvement in dermatomyositis was due to the administration of ruxolitinib rather than to the improvement in the hematologic cancer. We think that such a determination would help in better understanding the excellent response to this drug.

\section{Albert Selva-O'Callaghan, M.D., Ph.D. \\ Ernesto Trallero-Araguás, M.D., Ph.D. \\ Moises Labrador-Horrillo, M.D., Ph.D.}

Vall d'Hebron General Hospital

Barcelona, Spain

aselva@vhebron.net

No potential conflict of interest relevant to this letter was reported.

1. Hornung T, Janzen V, Wenzel J, Heidgen FJ, Wolf D, Bieber T. Remission of recalcitrant dermatomyositis treated with ruxolitinib. N Engl J Med 2014;371:2537-8.

2. Labrador-Horrillo M, Martínez MA, Selva-O'Callaghan A, et al. Anti-TIF1 $\gamma$ antibodies (anti-p155) in adult patients with dermatomyositis: comparison of different diagnostic assays. Ann Rheum Dis 2012;71:993-6.

3. Trallero-Araguás E, Rodrigo-Pendás JÁ, Selva-O'Callaghan A, et al. Usefulness of anti-p155 autoantibody for diagnosing cancer-associated dermatomyositis: a systematic review and meta-analysis. Arthritis Rheum 2012;64:523-32.

DOI: 10.1056/NEJMc1500688

THE AUTHORS REPLY: Dermatomyositis is known to be associated with cancers in approximately $25 \%$ of patients with the condition. However, dermatomyositis occurring in the course of myelofibrosis is extremely rare. Anti-TIF1 $\gamma$ antibodies have been linked to paraneoplastic dermatomyositis, but their clinical significance is still controversial, and the antibody level is not routinely measured. ${ }^{1}$

In our patient, we believe that the dramatic and rapid clinical effects were due to the antiinflammatory properties of the Janus kinase
(JAK) inhibitor, even though we cannot rule out the possibility that myelofibrosis-induced inflammation triggering dermatomyositis also might have been inhibited. Ruxolitinib induces only very limited activity against the JAK2-mutated clone, and its therapeutic effects in myelofibrosis are not determined by the patient's JAK2 mutational status, which supports the idea that clinical improvement is not due to a reduction in the tumor burden. ${ }^{2}$ Thus, inhibition of the production or secretion of cutaneous proinflammatory key cytokines (CXCL9 and CXCL10) appears to be the predominant therapeutic effect of the drug. ${ }^{3}$ This idea is further supported by the potent antiinflammatory capacity of ruxolitinib in graft-versus-host disease, a disorder in which the histologic and immunologic patterns show striking similarities to those of dermatomyositis. ${ }^{4,5}$

Thorsten Hornung, M.D.

Dominik Wolf, M.D.

Joerg Wenzel, M.D.

\section{University Hospital}

Bonn, Germany

joerg.wenzel@ukb.uni-bonn.de

Since publication of their letter, the authors report no further potential conflict of interest.

1. Fiorentino DF, Kuo K, Chung L, Zaba L, Li S, Casciola-Rosen L. Distinctive cutaneous and systemic features associated with antitranscriptional intermediary factor- $1 \gamma$ antibodies in adults with dermatomyositis. J Am Acad Dermatol 2015;72:449-55.

2. Guglielmelli P, Biamonte F, Rotunno G, et al. Impact of mutational status on outcomes in myelofibrosis patients treated with ruxolitinib in the COMFORT-II study. Blood 2014;123:215760.

3. Fridman JS, Scherle PA, Collins R, et al. Preclinical evaluation of local JAK1 and JAK2 inhibition in cutaneous inflammation. J Invest Dermatol 2011;131:1838-44.

4. Spoerl S, Mathew NR, Bscheider M, et al. Activity of therapeutic JAK 1/2 blockade in graft-versus-host disease. Blood 2014;123:3832-42

5. Wenzel J, Tüting T. An IFN-associated cytotoxic cellular immune response against viral, self-, or tumor antigens is a common pathogenetic feature in "interface dermatitis." J Invest Dermatol 2008;128:2392-402.

DOI: 10.1056/NEJMc1500688

\title{
Ebola Virus Disease among Children in West Africa
}

TO THE EDITOR: The epidemic of Ebola virus disease (EVD) in West Africa has caused clinical illness and deaths among persons with reported ages ranging from less than 1 year to more than 100 years. Most published estimates of key epidemiologic parameters have been based on patients of all ages ${ }^{1,2}$ and have thus been dominated by cases in which patients are 16 years of age or older, and as of January 5, 2015, these cases accounted for $79 \%$ of the confirmed and probable cases for which age has been reported.

Here we investigate the progression and out- 
come of EVD in confirmed and probable pediatric cases reported from Guinea, Liberia, and Sierra Leone, stratified according to age. The absolute and per capita case incidence of EVD among children younger than 16 years of age has been significantly and consistently lower than the incidence among adults in all three countries (Fig. 1A, 1B, and 1C). This pattern is similar to that observed in past EVD outbreaks. ${ }^{3,4}$ However, because the current epidemic is so large, it provides an opportunity to explore the ways in which epidemiologic and clinical parameters vary according to age. Although the age distribution of confirmed, probable, and suspected cases is similar in all three countries (Fig. S3 in the Supplementary Appendix, available with the full text of this letter at NEJM.org), the proportion of pediatric cases (those younger than 16 years of age) among all cases increased over the course of 2014 (Fig. 1C, and Fig. S4 in the Supplementary Appendix).

The mean incubation period (the average time from infection until symptom onset) was shortest, on average, in the youngest children, with means ranging from 6.9 days $(95 \%$ confidence interval [CI], 5.1 to 9.5) in 14 children younger than 1 year of age to 9.8 days (95\% CI, 8.7 to 11.1) in 184 children 10 to 15 years of age (Fig. 1D, and Table S1 and Fig. S5 in the Supplementary Appendix). Younger children also had shorter times from symptom onset to hospitalization and from symptom onset to death (Fig. 1D, and Fig. S6 and S7 and Tables S2 and S3 in the Supplementary Appendix). There was no clear evidence that age affected the distribution of the intervals between symptom onset and hospital discharge, between hospitalization and death, between hospitalization and hospital discharge, or between symptom onset and onward trans- mission (Fig. S8 to S11 and Tables S4 to S7 in the Supplementary Appendix).

Almost all children with EVD who were younger than 1 year of age had fever (92\%) before clinical presentation, and children younger than 16 years of age were more likely than adults to present with fever $(\mathrm{P}<0.001)$ (Table $\mathrm{S} 8$ and Fig. S13 in the Supplementary Appendix). Children were less likely than adults (i.e., persons 16 years of age or older) to report pain in the abdomen, chest, joints, or muscles, difficulty breathing or swallowing, and hiccups between symptom onset and clinical presentation $(\mathrm{P}<0.001)$; however, this finding may reflect the difficulty young children have in reporting such symptoms rather than a different symptom profile (Table S8 and Fig. S12 in the Supplementary Appendix). The case fatality rate (CFR) was lowest among children between 10 and 15 years of age and highest among those 4 years of age or younger (Fig. 1E, and Fig. S14 and S15 and Table S9 in the Supplementary Appendix). The CFR for persons younger than 45 years of age (most of whom are 5 to 44 years of age) was lower than that among those 45 years of age or older (Fig. 1E), a finding that is in line with that of an earlier report. ${ }^{5}$

The shorter incubation period in children, the relatively high risk of death among children younger than 5 years of age (as compared with older children), and the more rapid progression to death highlight the importance of including children among case contacts for follow-up, of examining children for early signs of disease during active case finding, and of explaining the risk of EVD to parents, guardians, and caregivers. All persons in whom EVD is suspected, but especially children, need the earliest possible referral for diagnostic testing, and children need age-appropriate treatment. The causes of the

Figure 1 (next page). Age-Group-Specific Incidence of Ebola Virus Disease in West Africa, Incubation Period, Intervals from Onset to Death and Onset to Hospitalization, and Case Fatality Rate.

Panel A shows the cumulative incidence of confirmed and probable cases of Ebola virus disease (EVD) according to age group and country. Panel B shows the cumulative incidence of confirmed and probable cases per 10,000 population according to age group and country. Panel $\mathrm{C}$ shows the overall age distribution for confirmed and probable cases according to month of symptom onset. Panel D shows the estimated average incubation period, the interval between symptom onset and death, and the interval between symptom onset and hospitalization according to age among persons with confirmed or probable EVD (with vertical lines indicating 95\% confidence intervals [Cls]). The numbers represent the sample sizes in each age group. Panel $E$ shows the estimated case fatality rate according to age among persons with confirmed or probable EVD (with $95 \% \mathrm{Cls}$ ), with representation of the total number of confirmed or probable cases of EVD cases in each age group and the total number of confirmed or probable cases of EVD for which there was information on the final outcome in each age group. 


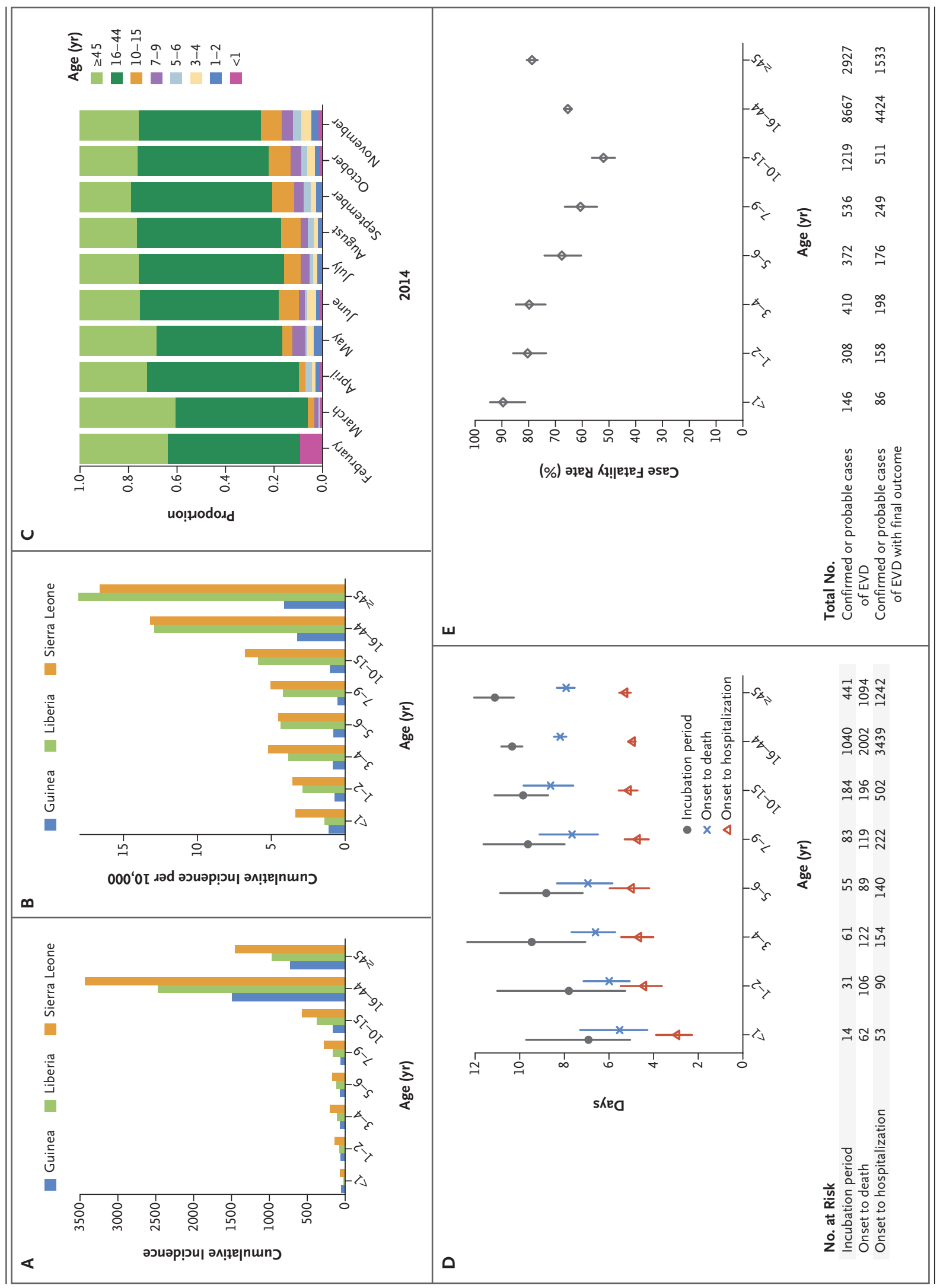


relatively rapid disease progression and relatively high CFR in the youngest children requires further investigation.

\section{WHO Ebola Response Team}

The authors (members of the WHO Ebola Response Team who contributed to this letter, in alphabetic order) are as follows: Junerlyn Agua-Agum, M.P.H., Archchun Ariyarajah, M.Sc., Isobel M. Blake, Ph.D., Anne Cori, Ph.D., Christl A. Donnelly, Sc.D., Ilaria Dorigatti, Ph.D., Christopher Dye, D.Phil., Tim Eckmanns, M.D., Neil M. Ferguson, D.Phil., Robert A. Fowler, M.D. C.M. M.S (Epi)., Christophe Fraser, Ph.D., Tini Garske, Ph.D., Wes Hinsley, Ph.D., Thibaut Jombart, Ph.D., Harriet L. Mills, Ph.D., Srinivas Murthy, M.D. C.M., Gemma Nedjati Gilani, Ph.D., Pierre Nouvellet, Ph.D., Louise Pelletier, M.D., Steven Riley, D.Phil., Dirk Schumacher, M.Sc., Anita Shah, M.Sc., Maria D. Van Kerkhove, Ph.D.

The authors' affiliations are as follows: World Health Organization (WHO), Geneva (J.A.-A., A.A., C.D., T.E., L.P., D.S., A.S.); Department of Medicine and Department of Critical Care Medicine, Sunnybrook Hospital, Interdepartmental Division of Critical Care Medicine, University of Toronto (R.A.F.); Department of Pediatrics, University of British Columbia (S.M.); The Medical Research Council Centre for Outbreak Analysis and Modelling, WHO Collaborating Centre for Infectious Disease Modelling, Department of Infectious Disease Epidemiology, Imperial College London, United Kingdom (I.M.B., A.C., C.A.D., I.D., N.M.F., C.F., T.G., W.H., T.J., H.L.M., G.N. G., P.N., S.R., M.D.V.K.); Robert Koch Institute, Department for Infectious Disease Epidemiology, Berlin, Germany (T.E., D.S.).

Address reprint requests to Dr. Donnelly (c.donnelly@imperial .ac.uk), Dr. Dye (dyec@who.int), or Dr. Ferguson (neil.ferguson@ imperial.ac.uk).

The study was conducted in support of the response to the Ebola outbreak in Guinea, Liberia, and Sierra Leone and is based on data routinely collected by national and international staff in conjunction with WHO.

Supported by the Medical Research Council, the Bill and Melinda Gates Foundation, the Models of Infectious Disease Agent Study of the National Institute of General Medical Sciences, National Institutes of Health, the Health Protection Research Units of the National Institute for Health Research, the European Union PREDEMICS consortium, the Wellcome Trust, and Fogarty International Center. Disclosure forms provided by the authors are available with the full text of this letter at NEJM.org.

1. WHO Ebola Response Team. Ebola virus disease in West Africa - the first 9 months of the epidemic and forward projections. N Engl J Med 2014;371:1481-95.

2. WHO Ebola Response Team. West African Ebola epidemic after one year - slowing but not yet under control. N Engl J Med 2015;372:584-7.

3. Outbreak of Ebola haemorrhagic fever, Uganda, August 2000-January 2001. Wkly Epidemiol Rec 2001;76:41-6.

4. Mupere E, Kaducu OF, Yoti Z. Ebola haemorrhagic fever among hospitalised children and adolescents in northern Uganda: epidemiologic and clinical observations. Afr Health Sci 2001; 1:60-5.

5. Bah EI, Lamah M-C, Fletcher T, et al. Clinical presentation of patients with Ebola virus disease in Conakry, Guinea. N Engl J Med 2015;372:40-7.

\section{DOI: $10.1056 /$ NEJMc1415318}

Correspondence Copyright (C) 2015 Massachusetts Medical Society.

\section{INSTRUCTIONS FOR LETTERS TO THE EDITOR}

Letters to the Editor are considered for publication, subject to editing and abridgment, provided they do not contain material that has been submitted or published elsewhere. Please note the following:

- Letters in reference to a Journal article must not exceed 175 words (excluding references) and must be received within 3 weeks after publication of the article.

- Letters not related to a Journal article must not exceed 400 words.

- A letter can have no more than five references and one figure or table.

- A letter can be signed by no more than three authors.

- Financial associations or other possible conflicts of interest must be disclosed. Disclosures will be published with the letters. (For authors of Journal articles who are responding to letters, we will only publish new relevant relationships that have developed since publication of the article.)

- Include your full mailing address, telephone number, fax number, and e-mail address with your letter.

- All letters must be submitted at authors. NEJM.org.

Letters that do not adhere to these instructions will not be considered. We will notify you when we have made a decision about possible publication. Letters regarding a recent Journal article may be shared with the authors of that article. We are unable to provide prepublication proofs. Submission of a letter constitutes permission for the Massachusetts Medical Society, its licensees, and its assignees to use it in the Journal's various print and electronic publications and in collections, revisions, and any other form or medium.

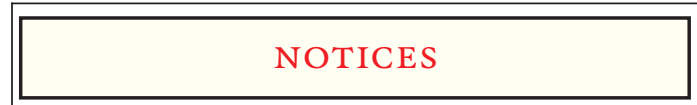

Notices submitted for publication should contain a mailing address and telephone number of a contact person or department. We regret that we are unable to publish all notices received. Notices also appear on the Journal's website (NEJM.org/medical-conference). The listings can be viewed in their entirety or filtered by specialty, location, or month.

\section{GYN SURGERY WEEK 2015}

The conference will be held in Fort Lauderdale, FL, April 15-18. It is presented by the Cleveland Clinic Women's Health Institute. Contact Cleveland Clinic Center for Continuing Education, 3050 Science Park Dr., AC313, Beachwood, Ohio 44122; or call (954) 659-5490; or e-mail cmeregistration@ccf.org; or see http://www.ccfcme.org/GYNSurgWeek.

\section{SURVIVAL SKILLS FOR TODAY'S GYNECOLOGIST 2015}

The annual meeting will be held in New York, April 17-19. Contact World Class CME, 6201 Fairview Rd., Suite 322, Charlotte, NC 28210; or call (980) 819-5095; or fax (980) 8195099; or email office@worldclasscme.com; or see http://www .worldclasscme.com/conferences/survival-skills-todays -gynecologist1.

\section{EUROPEAN MOLECULAR BIOLOGY LABORATORY}

The following meetings will be held in Heidelberg, Germany: "Mechanisms of Neurodegeneration" (June 14-17) and "Cancer Genomics" (Nov. 1-4).

Contact European Molecular Biology Laboratory, Course and Conference Office, Meyerhofstr. 1, D-69117 Heidelberg, Germany; or call (49) 6221387 8359; or fax (49) 6221387 8158; or see 